\title{
Dysarthrophonia in Association with Voice Analysis: A Case Report Govathi Nikhila K*
}

Institute of Neurosciences, Medanta-The Medicity, Gurgaon, India

\begin{abstract}
Stroke is the second leading cause of death worldwide and the brain damage caused by it can affect communication in several aspects. Voice analysis in dysarthria is challenging because of the complexity of the disorder and its effects on the speech production system. In this study we are presenting a 56-years-old male who was visited to Medanta Hospital with history of hypertension and chief complaint of Right upper limb weakness and slurred speech to the Emergency and later Clinically and Radio logically Diagnosed as LT MCA Infarct. Later, on the day 3 the patient has undergone Speech and Language Evaluation and Diagnosed with Spastic Dysarthria based on Frenched Dysarthria Assessment scale and later a detail Voice Analysis was done with using PRAAT software and analysed voice features. Voice analysis basically deals with decomposition of voice signal into voice parameters for processing the resulted features in desirable application. The features that are extracted in this paper are: frequency, pitch, voice intensity, formant, speech rate and pulse functions like Jitter (local), Jitter (local, absolute), Jitter (rap), Jitter (ppq5), Jitter (ddp), Shimmer (local), Shimmer (local, dB), Shimmer (apq3), Shimmer (apq5), Shimmer (apq11), Shimmer (dda) and Harmonic coefficients. Over all, we conclude with the voice parameters in spastic dysarthria which reveals interesting data on the voice quality with features which helps the clinician for better management. However, large sample study is required.
\end{abstract}

Keywords: Dysarthrophonia; Voice parameters; PRAAT software; Spastic dysarthria

\section{Introduction}

Dysarthrophonia is a combination of voice and speech disorder and it is a typical symptom of Parkinson's disease (PD) and occurs in most patients during the illness [1]. The progressive loss of the ability to communicate is an important source of disability in patients with PD. The typical pattern of hypokinetic dysarthria is characterized by a breathy or hoarse voice, reduced loudness and restricted pitch variability (monopitch and monoloudness), imprecise articulation and abnormalities of speech rate, and pause ratio. These multidimensional abnormalities of voice and speech have traditionally been attributed to the dopaminergic deficit manifesting in hypokinesia and rigidity of the laryngeal muscles (Figure 1).

The vocal analysis in individuals with the neurological disease can contribute toward reaching an early diagnosis and plays an important role in the follow-up as the disease progresses. In recent years, acoustic analysis of the voice in individuals with different neurological diseases is being carried out [2]. Acoustic analysis always complements perceptual evaluation and gives acoustic correlates for perceptual judgments in aspects of monopitch, pitch breaks, loudness, voice quality and dysarthria type.

\section{Dysarthria}

Dysarthria is a motor speech disorder. It results from impaired movement of the muscles used for speech production, including the lips, tongue, vocal folds, and/or diaphragm. The type and severity of dysarthria depend on which area of the nervous system is affected [3].

Causes: Stroke, Brain injury, Tumors, Parkinson's disease, Amyotrophic Lateral Sclerosis (ALS), Huntington's disease, Multiple sclerosis.

\section{Voice and its parameters}

Voice is the essential medium of man's communication in social as well as professional interactions. The human voice also reflects the state of health in many medical conditions which leads voice alterations in patients. The voice analysis is done by using PRAAT Software [4]. The vocal analysis in individuals with the neurological disease can contribute toward reaching an early diagnosis and plays an important role in the follow-up as the disease progresses [5]. Acoustic analysis always complements perceptual evaluation and gives acoustic correlates for perceptual judgments in aspects of monopitch, pitch breaks, loudness, voice quality, and dysarthria type. frequency, pitch, voice intensity, formant, speech rate and pulse functions like Jitter (local), Jitter (local, absolute), Jitter (rap), Jitter (ppq5), Jitter (ddp), Shimmer (local), Shimmer (local, dB), Shimmer (apq3), Shimmer (apq5), Shimmer (apq11), Shimmer (dda) and Harmonic coefficients (Figure 2).

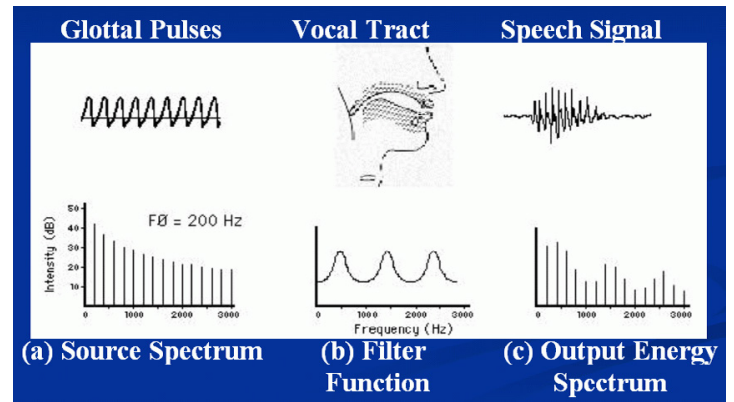

Figure 1: Acoustic analysis of the voice in individuals with different neurological diseases.

*Corresponding author: Govathi Nikhila K, Speech Language Pathologist Institute of Neurosciences, Medanta-The Medicity, Gurgaon, India, Tel: 9582972948; E-mail: gnikhilaks@gmail.com

Received November 14, 2017; Accepted September 03, 2018; Published September 06, 2018

Citation: Govathi Nikhila K (2018) Dysarthrophonia in Association with Voice Analysis: A Case Report. Brain Disord Ther 7: 247. doi: 10.4172/2168975X.1000247

Copyright: @ 2018 Govathi Nikhila K. This is an open-access article distributed under the terms of the Creative Commons Attribution License, which permits unrestricted use, distribution, and reproduction in any medium, provided the original author and source are credited. 


\section{Acoustic voice analysis}

It is done by speech/voice therapist and they evaluate voice quality by performing an acoustic voice analysis. It is also known as objective measurements. Recording of speech/voice are taken and acoustic parameters (pitch, loudness and range) are measured using sophisticated voice software. Like PRRAT which helps in analysing the voice parameters like, jitter, shimmer, pitch, loudness, brakes, etc. [6]. Normative data has been established for these acoustic measures and they are useful in defining the voice problem and in guiding voice therapy (Figure 3).

\section{Aim}

The aim of the study was to Analyse the speech and voice parameters by using PRAAT software in patient with spastic dysarthria in relation with before and after speech and voice therapy.

\section{Case Study}

A 56-years-old male reported to Medanta-The Medicity Hospital with stroke through Emergency ward with history of hypertension and diabetes mellitus [7]. Patient chief complaint was hypertension, right side weakness, facial deviation towards right side and mild weakness of left upper limb. Later immediate Medical Investigations was done, and the results as follows (Table 1):

Later, patient undergone immediate MRI and Radiological findings reveals Lt MCA Infarct and patient underwent with proper neurological management and Later on the day 3 of admission patient received detailed Speech and language Evaluation based on Frenched Dysarthria Screening, at bed side and diagnosed as "Spastic Dysarthria" with significant clinical and visual features like:

- Strain-Strangled voice quality, Hyper-nasality, Monotonous and Mono-loudness with pitch breaks present.

- Cough-moderate effort.

- Right side weakness, drooling and facial deviation, poor lingual, labial, and jaw co-ordination was noted.
- Reduced right palatal movement.

- Irregular breathing co-ordination,

- short breath.

- MPD- 4-5 secs.

- DDK-Reduced.

Later on, voice analysis was done by using PRAAT software to analyse the voice parameters. Voice parameters were elicited by using 4 different levels of speech/voice such as:

1. Phoneme level.

2. Word level (Standardised PB-Words).

3. Picture description and

4. General conversation.

\section{Results}

\section{Voice analysis before speech and voice therapy}

Phoneme level: A list of Phonemes was presented and asked the patient to repeat and voice/speech were recorded, and voice analysis was done by selecting an area to check the voice parameters at the level of phoneme (Table 2).

Word level: A list of Standard Words list (PB-Words) were presented and asked the patient to repeat and voice/speech were recorded, and voice analysis was done by selecting an area to check the voice parameters at word level (Table 3).

Picture description: A Standardised picture (Garden) were presented and asked the patient to describe the picture and voice/ speech were recorded and voice analysis was done by selecting an area to check the voice parameters at the level of simple sentences (Table 4).

General conversation: At this Level patient asked to describe about his life style and asked to describe about their family, favourite food, games and place, voice recording was done to analyse the voice parameters at the level of spontaneous speech (Table 5) [8-10].

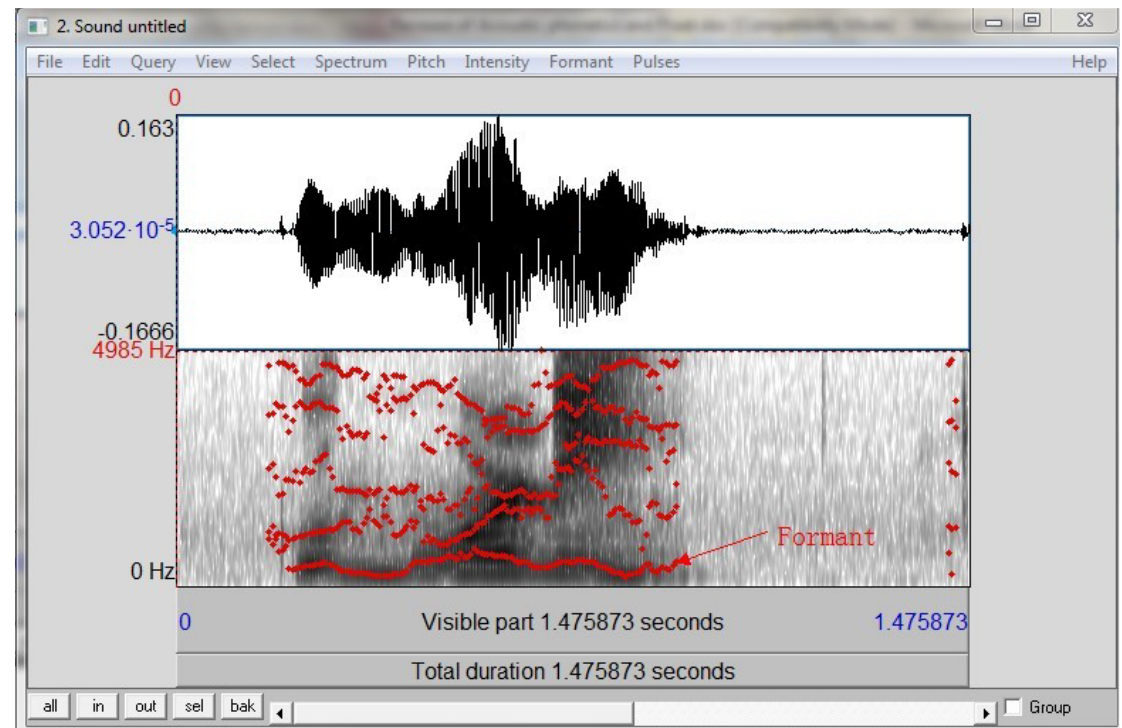

Figure 2: Acoustic analysis and perceptual evaluation of acoustic correlates in aspects of monopitch, pitch breaks, loudness, voice quality, and dysarthria type. 


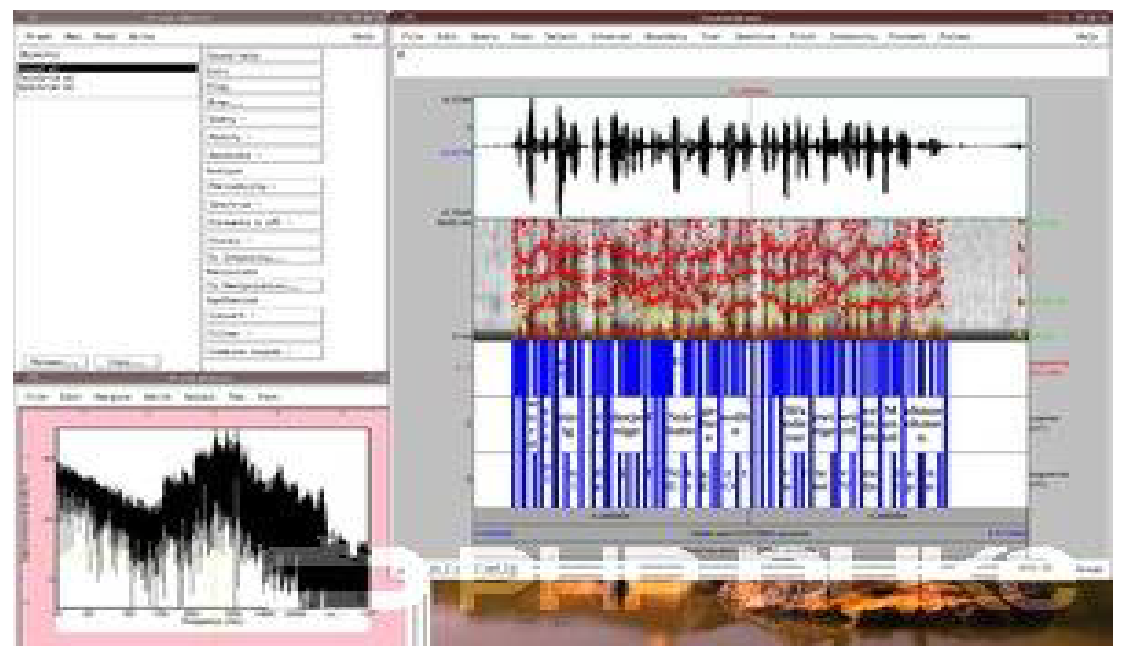

Figure 3: Analysis of the speech and voice parameters by using PRAAT software in patients with spastic dysarthria in relation with before and after speech and voice therapy.

\begin{tabular}{|c|c|c|}
\hline Risk factors & Physical systemic Examination & Neurological \\
\hline Diabetic: $y e s$ & BP: $200 / 110 \mathrm{~mm} \mathrm{Hg}$ & GCS: E4V5M6 \\
\hline Hypertension: Yes & Pulse: $80 / \mathrm{min}$ & CNS: Conscious, oriented EOM Full \\
\hline Alcoholic: No & RR: $20 / \mathrm{min}$ & speech slurred \\
\hline \multirow{3}{*}{ Smoker: No } & Chest: B/L-Clear & Left facial palsy present-UMN type \\
\cline { 2 - 3 } & CVS: S1, S2 Normal & No bell's phenomenon \\
\cline { 2 - 3 } & Abdomen: Soft, Non-tender & Power right upper and lower limb 5/5 \\
\cline { 2 - 3 } & Temp: Afebrile & Left upper limb 5/5 and lower limb 4/5. \\
\cline { 2 - 3 } & P/A: Soft non-tender &
\end{tabular}

Table 1: Medical investigations of the patient admitted with stroke reported to Medanta -The Medicity Hospital.

\begin{tabular}{|c|c|}
\hline Pitch: med- $128.091 \mathrm{~Hz}$ & Jitter rap-1.108 \% \\
\hline Mean-135.782 Hz & Jitter (ppq5)-1.073\% \\
\hline Min-117.279 Hz & Jitter (ddp)-3.325\% \\
\hline Max- $168.229 \mathrm{~Hz}$ & Shimmer: Local-14.8835\% \\
\hline Standard deviation- $16.079 \mathrm{~Hz}$. & Shimmer (local, db)-1.559 dB \\
\hline Pulses: No. of pulses-49 & Shimmer (apq3)-5.712 \% \\
\hline No. of periods- 48 & Shimmer (apq5)-8.325\% \\
\hline Mean period-7.393613E-3 Sec & Shimmer (apq11)-15.067\% \\
\hline Standard deviation of period- $0.853359 \mathrm{E}-3 \mathrm{Sec}$. & Shimmer (dda)-17.136\%. \\
\hline Voicing: Friction of locally unvoiced frames-34.909\% & Harmonicity of the voiced parts only: \\
\hline No. of voice breaks: 2 & Mean auto correlation- 0.856674 \\
\hline Degree of voice breaks: $28.300 \%$ & Mean Noise to Harmonics ratio- 0.184752 \\
\hline Jitter: Local- $2.224 \%$ & Mean Harmonics - to- Noise ratio- $8.709 \mathrm{~dB}$. \\
\hline Jitter local, absolute-64.445E-6 Sec & \\
\hline P/A: Soft non-tender & Left upper limb 5/5 and lower limb 4/5. \\
\hline
\end{tabular}

Table 2: Voice analysis done by selecting a particular area in order to check the voice parameters at the level of phoneme.

\section{Speech and voice therapy}

Later, immediate speech and voice therapy was recommended and started. Patient received Intensive speech and voice Therapy for 45 minutes for 6 consecutive days in a week [11-13]. Patient was trained with speech and voice techniques as follows (Table 6):

- Oro-Motor Exercises was recommended for 3-4 times a day each for 10 repetitions.

- Inhalation-Phonation
- Push-pull Techniques

- Tapping Exercise.

- Patient was recommended to follow the strategies throughout the day.

After 6 consecutive days of speech and voice therapy the patient underwent with voice analyses to analyse the voice parameters and to correlate the voice parameters before and after the speech and voice therapy. One level has been considered for voice analysis such as general conversation was done and again patient asked to describe about his life 
Citation: Govathi Nikhila K (2018) Dysarthrophonia in Association with Voice Analysis: A Case Report. Brain Disord Ther 7: 247. doi: 10.4172/2168975X.1000247

Page 4 of 6

\begin{tabular}{|c|c|}
\hline Pitch: med-125.758 Hz & Jitter (ppq5): 1.181\% \\
\hline Mean: $124.742 \mathrm{~Hz}$ & Jitter (ddp): $2.812 \%$ \\
\hline Min: $111.708 \mathrm{~Hz}$ & Shimmer: Local-10.935\% \\
\hline Max: $150.122 \mathrm{~Hz}$ & Shimmer (local, db): $1.020 \mathrm{~dB}$ \\
\hline Standard deviation: $6.274 \mathrm{~Hz}$. & Shimmer (apq3): $3.884 \%$ \\
\hline Pulses: No. of pulses: 124 & Shimmer (apq5): 6.550\% \\
\hline No. of periods: 120 & Shimmer (apq11): $14.277 \%$ \\
\hline Mean period: $8.026973 \mathrm{E}-6 \mathrm{Sec}$ & Shimmer (dda): $11.652 \%$ \\
\hline Standard deviation of period: $0.474873 \mathrm{E}-3 \mathrm{Sec}$. & Harmonicity of the voiced parts only: Mean auto correlation: 0.857308 \\
\hline Voicing: Friction of locally unvoiced frames: $49.302 \%$ & Mean Noise to Harmonics ratio: 0.222553 \\
\hline No. of voice breaks: 2 & Mean Harmonics to Noise ratio: $10.299 \mathrm{~dB}$ \\
\hline \multicolumn{2}{|l|}{ Degree of voice breaks: $16.840 \%$} \\
\hline \multicolumn{2}{|l|}{ Jitter: Local: $1.882 \%$} \\
\hline \multicolumn{2}{|l|}{ Jitter local, absolute: 151.061E-6 Sec } \\
\hline Jitter rap: $0.937 \%$ & \\
\hline
\end{tabular}

Table 3: Voice analysis done by selecting a particular area in order to check the voice parameters at the level of word level.

\begin{tabular}{|c|c|}
\hline Pitch: med-107.881 Hz & Jitter (ppq5)-1.475\% \\
\hline Mean-107.661 Hz & Jitter (ddp)-3.655\% \\
\hline Min-83.113 Hz & Shimmer: Local-12.636\% \\
\hline Max-135.584 Hz & Shimmer (local, db)-1.121 dB \\
\hline Standard deviation-9.428 Hz. & Shimmer (apq5)-7.172\% \\
\hline Pulses: No. of pulses-84 & Shimmer (apq11)-10.758\% \\
\hline No. of periods-80 & Shimmer (dda)-17.348\%. \\
\hline Mean period-9.304221E-3 Sec & Harmonicity of the voiced parts only: \\
\hline Standard deviation of period-0.798598E-3 Sec & Mean auto correlation-0.812149 \\
\hline Voicing: Friction of locally unvoiced frames-9.57\%. & Mean Noise to Harmonics ratio- 0.265690 \\
\hline Nogree of voice breaks-20.160\%. & Mean Harmonics - to- Noise ratio- $7.339 \mathrm{~dB}$. \\
\hline Jitter: Local- $2.707 \%$ & \\
\hline Jitter local, absolute-251.873E-6 Sec & \\
\hline Jitter rap-1.218\%. & \\
\hline
\end{tabular}

Table 4: Voice analysis done by selecting a particular area in order to check the voice parameters at the level of simple sentences.

\begin{tabular}{|c|c|}
\hline Pitch: med-115.993 Hz & Jitter (ppq5)-2.350\% \\
\hline Mean-125.169 Hz & Jitter (ddp)-5.447\% \\
\hline Min-90.527 Hz & Shimmer (local, db)-1.286 dB \\
\hline Max- $242.836 ~ H z$ & Shimmer (apq3)-6.203\% \\
\hline Standard deviation-29.400 Hz. & Shimmer (apq5)-10.455\% \\
\hline Pulses: No. of pulses-92 & Shimmer (apq11)-15.865\% \\
\hline No. of periods-88 & Shimmer (dda)-18.608\%. \\
\hline Standard deviation of period-1.551209E-3 Sec. & Harmonicity of the voiced parts only: Mean auto correlation-0.799342 \\
\hline Voicing: Friction of locally unvoiced frames-64.634\% & Mean Noise to Harmonics ratio- 0.309789 \\
\hline No. of voice breaks-2 & Mean Harmonics - to- Noise ratio- 7.239 dB \\
\hline Degree of voice breaks-22.118\%. & \\
\hline Jitter: Local- $3.578 \%$ & \\
\hline Jitter local, absolute-284.374E-6 Sec & \\
\hline Jitter rap-1.816\% & \\
\hline
\end{tabular}

Table 5: Voice analysis done by selecting a particular area in order to check the voice parameters at the level of spontaneous speech.

style and asked to describe about their family, favourite food, games and place, voice recording was done to analyse the voice parameters at the level of spontaneous speech [14,15]. Later, voice parameters were compared before and after speech and voice therapy and listed as below in Table 7.

\section{Discussion}

The perceptual analysis is the primary tool used by SLP to gather information about speech production characteristics of persons with various speech disorders. Acoustical analysis of the voice signal is one of the most useful methods to assess phonatory function or laryngeal pathology. There are very less articles published on Dysarthrophonia and among which one article only explains about the parameters of voice along with speech characteristics in stroke patient and that article was took as the base for this study and Cater G J in 1963 stated that it is a noninvasive method and provides objective and quantitative 
Citation: Govathi Nikhila K (2018) Dysarthrophonia in Association with Voice Analysis: A Case Report. Brain Disord Ther 7: 247. doi: 10.4172/2168975X.1000247

Page 5 of 6

\begin{tabular}{|c|c|}
\hline Pitch: med-111.846 Hz & Jitter (ppq5)-1.532\% \\
\hline Mean-12.006 Hz & Jitter (ddp)-4.215\% \\
\hline Min-88.649 Hz & Shimmer: Local-14.917\% \\
\hline Max-264.610 Hz & Shimmer (apq3)-5.956\% \\
\hline Standard deviation-34.527 Hz. & Shimmer (apq5)-9.535\% \\
\hline Pulses: No. of pulses-95 & Shimmer (apq11)-15.977\% \\
\hline No. of periods-92 & Shimmer (dda)-17.867\%. \\
\hline Mean period-8.349073E-3 Sec & Harmonicity of the voiced parts only: Mean auto correlation-0.730283 \\
\hline Standard deviation of period-1.928755E-3 Secs. & Mean Noise to Harmonics ratio- 0.434479 \\
\hline Voicing: Friction of locally unvoiced frames-16.667\%. & Mean Harmonics - to- Noise ratio- 5.197 dB \\
\hline No. of voice breaks-2 & \\
\hline Jitter local, absolute-264.074E-6 Sec & \\
\hline Jitter rap-1.405\% & \\
\hline
\end{tabular}

Table 6: Voice analysis done by selecting a particular area in order to check the voice parameters at the level of intensive speech and voice therapy.

\begin{tabular}{|c|c|c|}
\hline Voice parameters & Before Speech Therapy & After Speech Therapy \\
\hline \multicolumn{3}{|c|}{ Pitch } \\
\hline Med & $115.993 \mathrm{~Hz}$ & $111.846 \mathrm{~Hz}$ \\
\hline Mean & $125.169 \mathrm{~Hz}$ & $12.006 \mathrm{~Hz}$ \\
\hline Min & $90.527 \mathrm{~Hz}$ & $88.649 \mathrm{~Hz}$ \\
\hline Max & $242.836 \mathrm{~Hz}$ & $264.610 \mathrm{~Hz}$ \\
\hline Standard deviation & $29.400 \mathrm{~Hz}$ & $34.527 \mathrm{~Hz}$. \\
\hline \multicolumn{3}{|c|}{ Pulses } \\
\hline No. of pulses & 92 & 95 \\
\hline No. of periods & 88 & 92 \\
\hline Mean period & 7.947115E-3 Sec & 8.349073E-3 Sec \\
\hline Standard deviation of period & 1.551209E-3 Sec & 1.928755E-3 Sec \\
\hline \multicolumn{3}{|c|}{ Voicing } \\
\hline Friction of locally unvoiced frames & $64.634 \%$ & $16.667 \%$ \\
\hline No. of voice breaks & 2 & 2 \\
\hline Degree of voice breaks & $22.12 \%$ & $21.565 \%$ \\
\hline \multicolumn{3}{|c|}{ Jitter } \\
\hline Local- & $3.58 \%$ & $3.16 \%$ \\
\hline Jitter local, absolute & 284.374E-6 Sec & 264.074E-6 Sec \\
\hline Jitter rap & $1.82 \%$ & $1.41 \%$ \\
\hline Jitter (ppq5) & $2.35 \%$ & $1.53 \%$ \\
\hline Jitter (ddp) & $5.45 \%$ & $4.22 \%$ \\
\hline \multicolumn{3}{|c|}{ Shimmer } \\
\hline Local- & $15.20 \%$ & $14.92 \%$ \\
\hline Shimmer (local, db) & $1.286 \mathrm{~dB}$ & $1.533 \mathrm{~dB}$ \\
\hline Shimmer (apq3) & $6.20 \%$ & $5.96 \%$ \\
\hline Shimmer (apq5) & $10.46 \%$ & $9.54 \%$ \\
\hline Shimmer (apq11) & $15.87 \%$ & $15.98 \%$ \\
\hline Shimmer (dda) & $18.608 \%$ & $17.867 \%$ \\
\hline \multicolumn{3}{|c|}{ Harmonicity of the voiced parts only } \\
\hline Mean auto correlation & 0.799342 & 0.730283 \\
\hline Mean Noise to Harmonics ratio & 0.309789 & 0.434479 \\
\hline Mean Harmonics to Noise ratio & $7.239 \mathrm{~dB}$ & $5.197 \mathrm{~dB}$ \\
\hline
\end{tabular}

Table 7: Voice parameters compared before and after speech and voice therapy.

data. Acoustic parameters such as fundamental frequency (F0), vocal intensity, perturbation of the frequency (jitter), perturbation of amplitude (shimmer), harmonic-to-noise ratio, and maximum phonation frequency range helps to identify the physiological changes of the laryngeal system. Dysarthria results in abnormal values for several parameters in PRAAT, including absolute jitter (Jita), percentage jitter (Jitt), relative average perturbation, pitch perturbation quotient
(PPQ), smoothed PPQ, fundamental frequency variation, shimmer in $\mathrm{dB}$, shimmer percent, amplitude perturbation quotient, smoothed amplitude perturbation quotient, peak amplitude variation. By considering his statement and results into consideration I also analyzed the speech characteristics and voice parameters in stroke patient before and after speech and voice therapy and there by in this study minor differences were noted in voice parameters like pitch, jitter, shimmer, 
harmonic to noise ratio after 6 intensive speech and voice therapy sessions.

\section{Conclusion}

As this is a single case study with a less duration period a detail investigations couldn't be done. Further, researcher can focus on this aspects. However large population study is required. Over all, we conclude with the voice parameters in spastic dysarthria which reveals interesting data on the voice quality with features which helps the clinician for better management. Based on the information obtained on the perceptual-acoustic analysis of the client's voice, we conclude that voice analysis is a useful tool in the identification of voice problems in dysarthria and can help the voice specialist focus on appropriate aspects in the intervention of these individuals.

\section{References}

1. Ho AK, lansek R, Marigliani C, Bradshaw JL, Gates S (1999) Speech impairment in a large sample of patients with Parkinson's disease. Behavioural neurology 11: 131-137.

2. Perez-Lloret $S$, Nègre-Pagès $L$, Ojero-Senard $A$, Damier $P$, Destee $A$, et al (2012) Oro-buccal symptoms (dysphagia, dysarthria, and sialorrhea) in patients with Parkinson's disease: preliminary analysis from the French COPARK cohort. Eur J Neurol 19: 28-37.

3. Miller N, Allcock L, Jones D, Noble E, Hildreth AJ, et al. (2007) Prevalence and pattern of perceived intelligibility changes in Parkinson's disease. J Neurol Neurosurg Psychiatry 78: 1188-1190.

4. Dowding $\mathrm{CH}$, Shenton $\mathrm{CL}$, Salek SS (2006) A review of the health-related quality of life and economic impact of Parkinson's disease. Drugs Aging 23 693-721.

5. Miller N, Noble E, Jones D, Allcock L, Burn DJ (2008) How do I sound to me? Perceived changes in communication in Parkinson's disease. Clinical Rehabilitation 22: 14-22.

6. Miller N, Noble E, Jones D, Burn D (2006) Life with communication changes in Parkinson's disease. Age Ageing 35: 235-239.

7. Shirvan RA, Tahami E (2011) Voice analysis for detecting Parkinson's disease using genetic algorithm and KNN classification method. In: $18^{\text {th }}$ Iranian Conference on Biomedical Engineering 1416: 278-283.

8. Jankovic J (2008) Parkinson's disease: Clinical features and diagnosis. J Neurol Neurosurg Psychiatry 79: 368-376.

9. Lang AE, Lozano AM (1998) Parkinson's disease. N Engl J Med 339: 1130-1143.

10. Dauer W, Przedborski S (2003) Parkinson's disease: Mechanisms and models. Neuron 39: 889-909.

11. Waters $\mathrm{CH}$ (2008) Diagnosis and management of Parkinson's disease. Professional Communications 2: 8.

12. Duffy JR. (2005) Motor speech disorders: Substrates, differential diagnosis, and management. (2nd edn). St Louis, MO: Mosby, USA.

13. Huber JE, Stathopoulos ET, Ramig LO, Lancaster SL (2003) Respiratory function and variability in individuals with Parkinson disease: Pre-and post-Lee Silverman voice treatment. J Med Speech-Language Path 11: 185-202.

14. Theodoros DG, Murdoch BE, Thompson EC (1995) Hyper-nasality in Parkinson's disease: A perceptual and physiological analysis. J Med SpeechLanguage Path 3: 73-84

15. Canter GJ (1963) Speech characteristics of patients with Parkinson's disease: I. Intensity, pitch, and duration. J Speech Hear Disord 28: 221-229. 